\begin{tabular}{|c|c|}
\hline Title & Guided Modes of Nonlinear Slot Waveguides \\
\hline Author(s) & Fujisawa, T.; Koshiba, M. \\
\hline Citation & $\begin{array}{l}\text { IEEE Photonics Technology Letters, 18(14), 1530-1532 } \\
\text { https://doi.org/10.1109/LP .2006.877593 }\end{array}$ \\
\hline Issue Date & $2006-07$ \\
\hline Doc URL & http:/hdl.handle.net/2115/44742 \\
\hline Rights & $\begin{array}{l}\text { @2006 IEEE. Personal use of this material is permitted. However, permission to reprint/republish this material for } \\
\text { advertising or promotional purposes or for creating new collective works for resale or redistribution to servers or lists, } \\
\text { or to reuse any copyrighted component of this work in other works must be obtained from the IEEE. }\end{array}$ \\
\hline Type & article \\
\hline File Information & CPL427-4-6.pdf \\
\hline
\end{tabular}

Instructions for use 


\title{
Guided Modes of Nonlinear Slot Waveguides
}

\author{
T. Fujisawa, Member, IEEE, and M. Koshiba, Fellow, IEEE
}

\begin{abstract}
Guided modes of nonlinear slot waveguides are investigated for the first time and their unusual modal properties are demonstrated. Nonlinear phase shifts of slot waveguides induced by optical Kerr effect exhibit saturable behavior for strong optical intensity. This is because a self-localization effect of electromagnetic fields induced by optical Kerr effect is compensated with a field broadening originating from a unique confinement mechanism of slot waveguides. Nonlinear phase shifts on various structural parameters are thoroughly investigated. All the results presented in this letter are obtained by using a rigorous full-vector finite-element method to ensure accurate analysis.
\end{abstract}

Index Terms-Finite-element method (FEM), nonlinear optical waveguide, optical Kerr effect, slot waveguide.

\section{INTRODUCTION}

$\mathbf{O}$ PTICAL waveguide devices utilizing nonlinear optical effects are promising candidates for the components of future telecommunication networks because of their applicability to all-optical signal processing devices. Among various nonlinear optical effects, the optical Kerr effect has attracted considerable attention because of its ultrafast response time and is widely used for a lot of applications. Due to the recent advancement in microstructure fabrication technology, wavelength scale optical waveguides have been fabricated such as $\mathrm{Si}$-wire and photonic crystal waveguides for ultrasmall optical integrated circuits. In these waveguides, the intensity of light is significantly larger than that of conventional low-index-contrast optical waveguides leading to the dramatic enhancement of nonlinear optical effect. A lot of research on nonlinear optical devices based on these microstructured waveguides has been done [1]-[7]. Recently, so-called slot waveguides have been proposed and fabricated as alternative candidates for optical integrated circuits based on microstructure waveguides [8], [9]. In the slot waveguide, low-refractive-index material (slot region) is sandwiched by high-refractive-index materials (see Fig. 1). In such a waveguide geometry, the guided mode polarized to one of the major axes (in this letter, $x$ : quasi-TE modes) is strongly confined in the low-index slot region [8], [9] because of the electric field discontinuity between high refractive index and low refractive index materials, and light can be strongly confined in the low-index slot region by total internal reflection. The upper right and lower right insets in Fig. 1 show linear guided mode field distributions of the slot waveguide for quasi-TE and quasi-TM (the electric field is polarized to $y$ axis) modes, respectively. We can see that light confinement in the slot region is very strong for quasi-TE modes. Therefore, it is expected to enhance nonlinearity effectively and all-optical switching devices based on slot waveguides have been proposed [10], [11]. In these nonlinear

Manuscript received March 2, 2006; revised April 16, 2006.

The authors are with the Division of Media and Network Technologies, Hokkaido University, Sapporo 060-0814, Japan (e-mail: fujisawa@dpo7.ice. eng.hokudai.ac.jp; koshiba@ist.hokudai.ac.jp).

Digital Object Identifier 10.1109/LPT.2006.877593

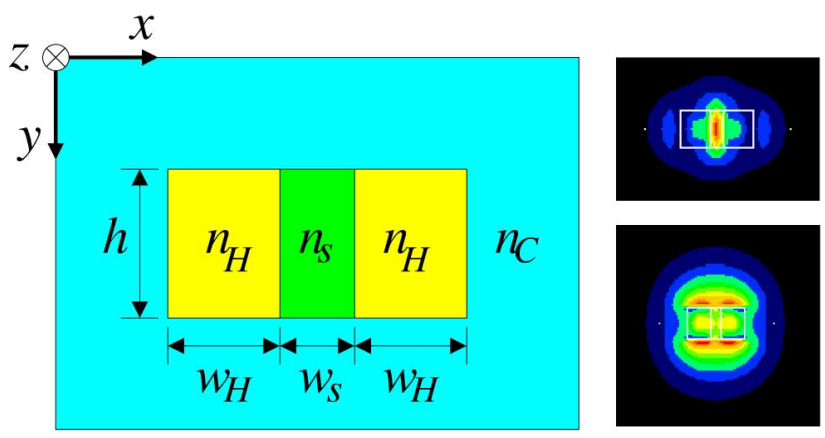

Fig. 1. Geometry of nonlinear slot waveguide. The upper right and lower right insets show the linear guided mode electric field distributions of the slot waveguide for quasi-TE and quasi-TM modes, respectively. (Color version available online at http://ieeexplore.ieee.org.)

optical switching devices, a refractive index change (or phase shift) induced by optical Kerr effect is utilized for switching operations, and important information for designing nonlinear optical devices, for example, nonlinear Mach-Zehnder interferometers [7]. To obtain the nonlinear phase shift accurately, the guided mode analysis of nonlinear optical waveguides through the rigorous numerical methods are necessary. However, intensity-dependent phase shifts in nonlinear slot waveguides have not been investigated so far.

In this letter, guided modes of nonlinear slot waveguides are investigated for the first time and their unusual modal properties are demonstrated. Nonlinear phase shifts of slot waveguides induced by optical Kerr effect exhibit saturable behavior for strong optical intensity. This is because a self-localization effect of electromagnetic fields induced by the optical Kerr effect is compensated with a field broadening originating from a unique confinement mechanism of slot waveguides. Nonlinear phase shifts on various structural parameters are thoroughly investigated. All the results presented in this letter are obtained by using a rigorous full-vector finite-element method (FEM) specially formulated for the analysis of nonlinear optical waveguides [12].

\section{GUIDED MODES OF NONLIEAR SLOT WAVEGUIDES}

Here, we consider a nonlinear slot waveguide as shown in Fig. 1. Waveguide parameters are as follows: The refractive index of high-index region and cladding are, respectively, taken as $n_{H}=3.48(\mathrm{Si})$ and $n_{C}=1.46\left(\mathrm{SiO}_{2}\right)$; the waveguide height $h=250 \mathrm{~nm}$; and $w_{H}=200 \mathrm{~nm}$. $w_{S}$ is the width of slot region. We assume that the material embedded in the slot region has Kerr-type nonlinearity, and its intensity-dependent refractive index $n_{S}$ is given by

$$
n_{S}=n_{L}\left(1+\frac{n_{2}|\boldsymbol{E}|^{2}}{Z_{0}}\right)^{1 / 2}
$$




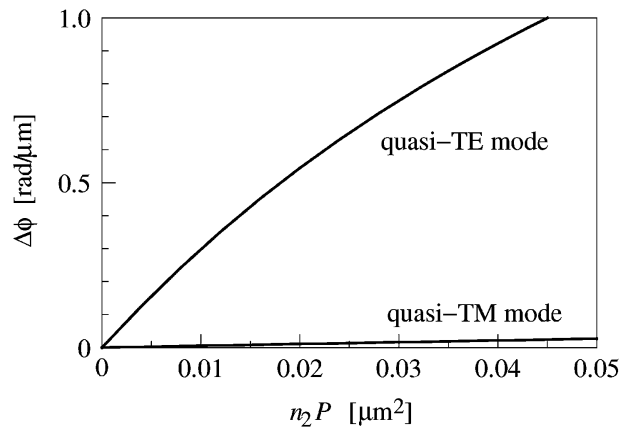

Fig. 2. Nonlinear phase shifts of a slot waveguide with $w_{S}=100 \mathrm{~nm}$ and $n_{L}=1.46$ as a function of $n_{2} P$.

where $n_{L}$ is the linear part of the refractive index, $n_{2}\left[\mathrm{~m}^{2} / \mathrm{W}\right]$ is the nonlinear coefficient, $\boldsymbol{E}$ is the electric field distributions, and $Z_{0}$ is the free-space impedance. One possible candidate for the nonlinear material is Si nanocrystals [10], [11] whose $n_{2}$ is four orders of magnitude larger than that of $\mathrm{SiO}_{2}$. The operating wavelength is assumed to be $1.55 \mu \mathrm{m}$. Ratios of optical power carried in the slot region for quasi-TE and TM modes are $26 \%$ and $9.4 \%$, respectively, showing the strong confinement for quasi-TE modes. Full-vector modal solver based on $\boldsymbol{E}$-field FEM [12] is utilized to obtain modal solution of nonlinear slot waveguides. Full-vector FEM is one of the most appropriate numerical methods for the analysis of slot waveguides because boundary conditions between different materials are automatically satisfied without special treatments. Fig. 2 shows the nonlinear phase shifts of a slot waveguide with $w_{S}=100 \mathrm{~nm}$ and $n_{L}=1.46$ as a function of $n_{2} P$, where $P$ is the optical power. Here, the nonlinear phase shift per unit length $\Delta \phi$ is defined as [4], [5]

$$
\Delta \phi=k_{0}\left(n_{\mathrm{eff}}^{N L}-n_{\mathrm{eff}}^{L}\right)
$$

where $k_{0}$ is the free-space wavenumber, $n_{\mathrm{eff}}^{N L}$ and $n_{\mathrm{eff}}^{L}$ are the nonlinear and linear effective refractive indexes, respectively. Here, the values of $n_{\text {eff }}^{L}$ for quasi-TE and TM modes are 1.729706 and 1.755922 , respectively. Nonlinear phase shifts for quasi-TE modes are significantly larger than those for quasi-TM modes because the field confinement in the slot region for quasi-TM modes is not so strong (see field distributions in Fig. 1). For quasi-TE modes, a saturable behavior for $\Delta \phi$ can be seen for larger values of $n_{2} P$. This is quite unusual because the nonlinear refractive index is proportional to optical intensity as can be seen in (1). The behavior can be explained as follows. For smaller values of $n_{2} P$, the refractive index change in the slot region is not so large resulting in linear increase of $\Delta \phi$. For larger values of $n_{2} P$, because the refractive index change in the slot region becomes large, refractive-index-contrast between $n_{S}$ and $n_{H}$ is reduced. Because the degree of light confinement in the slot region becomes weaker for lower index contrast between $n_{S}$ and $n_{H}$, the field is broadened for larger values of $n_{2} P$ resulting in weaker nonlinearity. Therefore, contributions to the field confinement from optical Kerr effect and the slot waveguide confinement are balanced each other resulting in the saturable behavior for strong optical intensity. This is also confirmed by effective area $A_{\text {eff }}$ as shown in Fig. 3 . For smaller optical powers, $A_{\text {eff }}$ is linearly decreased with $n_{2} P$ because the

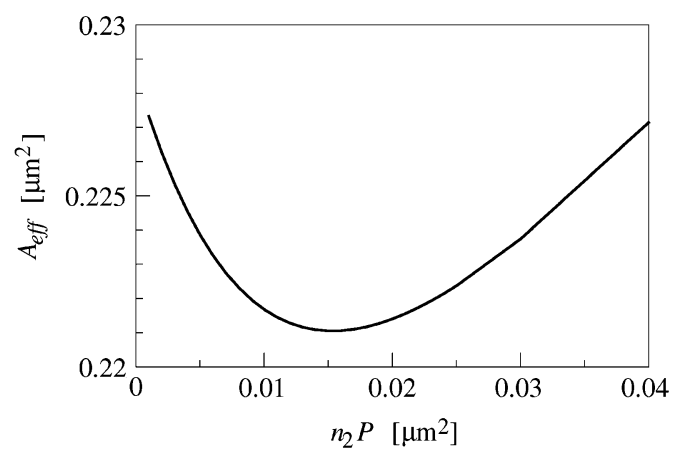

Fig. 3. Effective area of the nonlinear slot waveguide with $w_{S}=100 \mathrm{~nm}$ and $n_{L}=1.46$ for quasi-TE modes as a function of $n_{2} P$.

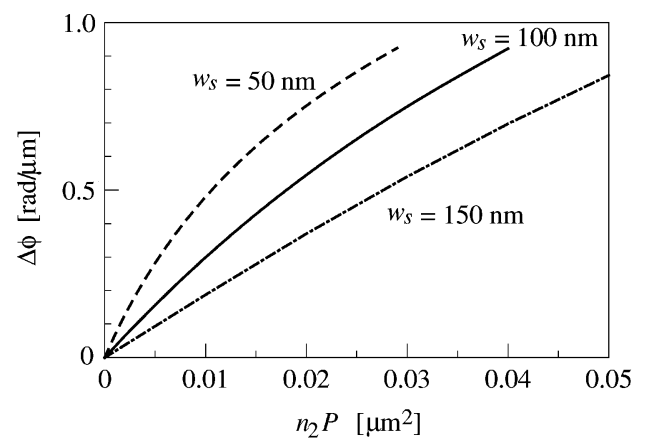

Fig. 4. Nonlinear phase shifts of slot waveguides with $n_{L}=1.46$ as a function of $n_{2} P$ for different values of $w_{S}$.

field is more confined to the slot region due to the optical Kerr effect. When the optical power is further increased, the value of $A_{\mathrm{eff}}$ becomes larger due to the field broadening originating from reduced index contrast between $n_{S}$ and $n_{H}$. Hereafter, we focus on the nonlinear phase shift for quasi-TE modes. Fig. 4 shows nonlinear phase shifts of slot waveguides as a function of $n_{2} P$ with $n_{L}=1.46$ for different values of $w_{S}$. The values of $\Delta \phi$ are larger for $w_{S}=50 \mathrm{~nm}$ because the intensity in the slot region becomes stronger for smaller values of $w_{S}$. Fig. 5(a) shows nonlinear phase shifts of slot waveguides as a function of $n_{2} P$ for different values of $n_{L}$. By increasing the value of $n_{L}$, nonlinear phase shifts become larger because nonlinear refractive index change is proportional to $n_{L}$ as can be seen in (1). However, for $n_{L}=1.8, \Delta \phi$ is almost unchanged compared with that for $n_{L}=1.46$ because for larger values of $n_{L}$; a light intensity in the slot region becomes smaller resulting in weaker nonlinearity, as shown in Fig. 5(b), which show linear guided mode field distributions at the center of the waveguides in the $x$ direction. Because light intensities in the slot region for $n_{L}=1.3$ and 1.46 are almost the same, $\Delta \phi$ for $n_{L}=1.46$ is larger than that for $n_{L}=1.3$. For $n_{L}=1.8$, the light intensity in the slot region is lower than that for $n_{L}=1.46$ due to the low-index-contrast between $n_{S}$ and $n_{H}$, and thus, the nonlinearity is weakened leading to smaller increase in $\Delta \phi$. Therefore, to effectively enhance the nonlinearity, the linear refractive index of nonlinear materials is very important. Such characteristics cannot be seen in usual nonlinear channel optical waveguides. Fig. 6 shows the nonlinear phase shifts of slot waveguides with $w_{S}=100 \mathrm{~nm}$ and $n_{L}=1.46$ as a function of $n_{2} P$ for different wavelengths. Because the confinement 


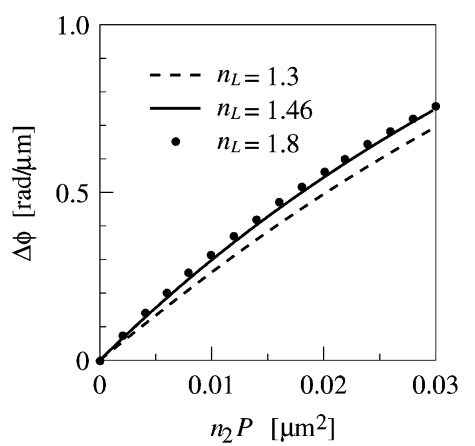

(a)

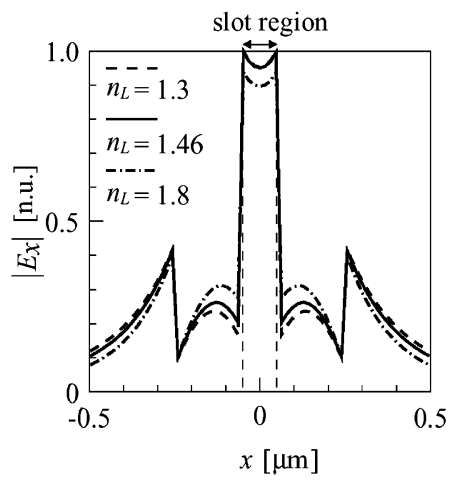

(b)

Fig. 5. (a) Nonlinear phase shifts of slot waveguides with $w_{S}=100 \mathrm{~nm}$ as a function of $n_{2} P$ for different values of $n_{L}$. (b) Linear guided mode field distributions of slot waveguides at the center of the waveguides in $x$ direction.

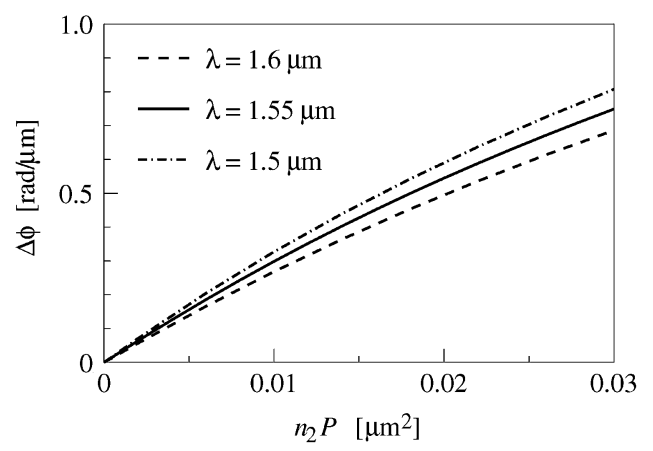

Fig. 6. Nonlinear phase shifts of slot waveguides with $w_{S}=100 \mathrm{~nm}$ and $n_{L}=1.46$ as a function of $n_{2} P$ for different wavelengths.

mechanism of slot waveguides is not resonant effect [8], the modal properties are not so sensitive to wavelength.

\section{CONCLUSION}

We have investigated nonlinear modal properties of slot waveguides by using rigorous full-vector FEM. From the numerical results presented here, it was shown that for strong optical intensity, the saturable behavior can be seen in the nonlinear phase shift. The behavior stems from a cancellation of self-localization of the electromagnetic field due to the optical Kerr effect by the field broadening induced by the slot waveguide confinement. It was also pointed out that to effectively enhance the nonlinearity in slot waveguides, the linear refractive index of nonlinear material is important as well as the nonlinear coefficient.

\section{REFERENCES}

[1] T. Fujisawa and M. Koshiba, "Time-domain beam propagation method for nonlinear optical propagation analysis and its application to photonic crystal circuits," J. Lightw. Technol., vol. 22, no. 2, pp. 684-691, Feb. 2004.

[2] V. R. Almeida, C. A. Barrios, P. R. Panepucci, M. Lipson, M. A. Foster, D. G. Ouzounov, and A. L. Gaeta, "All-optical switching on a silicon chip," Opt. Lett., vol. 29, pp. 2867-2869, Dec. 2004.

[3] T. Tsuchizawa, K. Yamada, H. Fukuda, T. Watanabe, J. Takahashi, M. Takahashi, T. Shoji, E. Tamechika, S. Itabashi, and H. Morita, "Microphotonics devices based on silicon micro fabrication technology," IEEE J. Sel. Topics Quantum Electron., vol. 11, no. 1, pp. 232-240, Jan./Feb. 2005.

[4] T. Fujisawa and M. Koshiba, "Finite-element mode-solver for nonlinear periodic optical waveguides and its application to photonic crystal circuits," J. Lightw. Technol., vol. 23, no. 1, pp. 382-387, Jan. 2005.

[5] R. L. Espinola, J. I. Dadap, R. M. Osgood, Jr., S. J. McNab, and Y. A. Vlasov, " $C$-band wavelength conversion in silicon photonic wire waveguides," Opt. Express, vol. 13, pp. 4341-4349, May 2005.

[6] Q. Xu, V. R. Almeida, and M. Lipson, "Micrometer-scale all-optical wavelength converter on silicon," Opt. Lett., vol. 30, pp. 2733-2735, Oct. 2005.

[7] T. Fujisawa and M. Koshiba, "Finite-element modeling of nonlinear Mach-Zehnder interferometers based on photonic crystal waveguides for all-optical signal processing," J. Lightw. Technol., vol. 24, no. 1, pp. 617-623, Jan. 2006.

[8] V. R. Almeida, Q. Xu, C. A. Barrios, and M. Lipson, "Guiding and confining light in void nanostructures," Opt. Lett., vol. 29, pp. 1209-1211, Jun. 2004.

[9] Q. Xu, V. R. Almeida, R. R. Panepucci, and M. Lipson, "Experimental demonstration of guiding and confining light in nanometer-size lowrefractive-indx material," Opt. Lett., vol. 29, pp. 1626-1628, Jul. 2004.

[10] C.A. Barrios, "High-performance all-optical silicon microswitch," Electron. Lett., vol. 40, pp. 862-863, Jul. 2004.

[11] T. Fujisawa and M. Koshiba, "All-optical logic gates based on nonlinear slot waveguide coupler," J. Opt. Soc. Amer. B, vol. 23, pp. 684-691, Apr. 2006.

[12] T. Fujisawa and M. Koshiba, "Finite element characterization of chromatic dispersion in nonlinear holey fibers," Opt. Express, vol. 11, pp. 1481-1489, Jun. 2003. 